\title{
The Poisson Variation of Montmort's Matching Problem
}

\author{
DON RAWLINGS \\ California Polytechnic State University \\ San Luis Obispo, CA 93407
}

The matching problem In 1708, Pierre Rémond de Montmort [6] proposed and solved the following problem:

Matching problem From the top of a shuffled deck of $n$ cards having face values $1,2, \ldots, n$, cards are drawn one at a time. A match occurs if the face value on a card coincides with the order in which it is drawn. For instance, if the face values of a five-card deck appear in the order 25341 , then there are two matches ( 3 and 4$)$. What is the probability that no match occurs?

Known as le problème des rencontres, Montmort's problem has become a classic. Treated in almost every modern text on combinatorics, it has been recast in a variety of amusing guises including the random switching of $n$ hats by a possibly tipsy cloakroom attendant, the random placement of $n$ letters in $n$ envelopes by a secretary apparently having a bad day, and even in terms of a wager on the ordering in which the horses finish in a given race (for instance, see [3], [4], and [8]).

As is well known (e.g., see [2, p. 107]), the principle of inclusion-exclusion may be used to show that the probability $M_{n, k}$ of $k$ matches occurring with an $n$-card deck is

$$
M_{n, k}=\frac{1}{k !} \sum_{j=0}^{n-k} \frac{(-1)^{j}}{j !} .
$$

For $k=0$, of course, (1) gives the solution to Montmort's problem. The celebrated fact that the probability of no match occurring is asymptotic to $e^{-1}$, that is,

$$
\lim _{n \rightarrow \infty} M_{n, 0}=e^{-1}
$$

also follows from (1). Incidentally, the distribution of matches $\left\{M_{n, k}\right\}_{0 \leq k \leq n}$ has applications in psychology and in testing psychic powers (e.g., see [1], [2, p. 108], or [9]).

The Poisson variation: marking the cards The following twist, henceforth referred to as the Poisson variation, is added to Montmort's problem: Suppose that as each card is drawn it is marked (by underlining its face value) with probability $(1-t)$ where $t$ is a real number between 0 and 1 . As underlined integers are evidently distinct from ordinary ones, they cannot be involved in a match. So, if from a five-card deck the sequence of marked and unmarked face values $125 \underline{4} 3$ appear, then the only match involves 2.

Perhaps odd at first glance, this twist gives rise to some aesthetically pleasing mathematics. To be precise, it leads to the following natural extensions of (1) and (2) from $t=1$ to the entire interval $[0,1]$. 
THEOREM 1. The probability $P_{n, k}$ of $k$ matches occurring in the Poisson variation of the matching problem with an n-card deck is given by

$$
P_{n, k}=\frac{t^{k}}{k !} \sum_{j=0}^{n-k} \frac{(-1)^{j} t^{j}}{j !}
$$

Moreover, $\left\{P_{n, k}\right\}_{0 \leq k \leq n}$ is asymptotically Poisson with parameter $t$ :

$$
\lim _{n \rightarrow \infty} P_{n, k}=\frac{t^{k}}{k !} e^{-t}
$$

So the asymptotic probability of no matches occurring is now $e^{-t}$ !

Proof. To formalize matters, let $\Omega_{n}$ denote the set generated by first randomly permuting the integers 1 through $n$ and then underlining each integer with probability $(1-t)$ where $0 \leq t \leq 1$. The probability of $w=\underline{2} 4 \underline{1} \underline{3} \in \Omega_{4}$ being generated is therefore $t(1-t)^{3} / 4$ !. For $t=1$, note that $\Omega_{n}$ coincides with the set of permutations $S_{n}$ of $\{1,2, \ldots, n\}$.

A fixed point of $w=w_{1} w_{2} \ldots w_{n} \in \Omega_{n}$ is an integer $j$ satisfying $w_{j}=j$. For example, $w=\underline{1} 25 \underline{4} 3 \in \Omega_{5}$ has one fixed point $(j=2)$. Furthermore, an element in $\Omega_{n}$ having no fixed points is said to be a derangement.

Obviously, a sequence of marked and unmarked face values appearing in the Poisson variation of the matching problem may be naturally identified with an element of $\Omega_{n}$. Moreover, under this identification, matches correspond to fixed points and the event of no matches evidently coincides with a derangement.

Let $F_{n, k}$ be the number of permutations in $S_{n}$ having $k$ fixed points. Making the usual appeal that such a permutation may be constructed by selecting $k$ integers to fix and then "deranging" the remaining $(n-k)$ integers gives the well known identity $F_{n, k}=\left(\begin{array}{l}n \\ k\end{array}\right) F_{n-k, 0}$.

As $M_{n, k}=F_{n, k} / n !$, it follows that

$$
M_{n, k}=\frac{F_{n-k, 0}}{(n-k) ! k !} .
$$

Since $\left\{M_{n, k}\right\}_{0 \leq k \leq n}$ is a probability distribution, we also have

$$
\sum_{k=0}^{n} M_{n, k}=1 .
$$

An element in $\Omega_{n}$ having $k$ fixed points may be generated by first selecting a permutation $\sigma \in S_{n}$ with $l$ fixed points where $k \leq l \leq n$ and then underlining $(l-k)$ of $\sigma$ 's fixed points. From the properties of elementary probability, (3), and the binomial theorem, it follows that

$$
\begin{aligned}
P_{n, k} & =\sum_{l=k}^{n} M_{n, l}\left(\begin{array}{l}
l \\
k
\end{array}\right) t^{k}(1-t)^{l-k}=\sum_{l=k}^{n} \frac{F_{n-l, 0}}{l !(n-l) !}\left(\begin{array}{l}
l \\
k
\end{array}\right) t^{k} \sum_{j=0}^{l-k}(-1)^{j}\left(\begin{array}{c}
l-k \\
j
\end{array}\right) t^{j} \\
& =\frac{t^{k}}{k !} \sum_{j=0}^{n-k} \frac{(-1)^{j} t^{j}}{j !} \sum_{l=j+k}^{n} \frac{F_{n-l, 0}}{(n-l) !(l-k-j) !}
\end{aligned}
$$


To complete the proof, just note that an index shift and (4) imply that the rightmost sum at the end of (5) is

$$
\sum_{\nu=0}^{n-k-j} \frac{F_{n-k-j-\nu, 0}}{(n-k-j-\nu) ! \nu !}=\sum_{\nu=0}^{n-k-j} M_{n-k-j, \nu}=1 .
$$

Concluding remarks Montmort's interest in the matching problem was motivated by the more colorful and complicated problem of determining the banker's advantage in a gambling game known as Treize. A detailed account of Treize and of the work of Montmort and Nicholas Bernoulli on the matching problem is given in [5]. Montmort's problem has been extended in a variety of other ways. In this regard, the articles [1], [3], [7], and [8] are highly recommended. The question of whether marking cards provides nice extensions of the results in [1], [3], and [8] is left open.

Finally, Theorem 1 may be deduced from a result in [7] that involves Bernoulli trials, partitions, and $q$-combinatorics. The preceding proof is far less encumbered.

\section{REFERENCES}

1. D. E. Barton, The matching distributions: Poisson limiting forms and derived methods of approximation, J. Royal Stat. Soc. 20 (1958), 73-92.

2. W. Feller, An Introduction to Probability Theory and Its Applications, Vol. I, John Wiley and Sons, New York, NY, 1990.

$\rightarrow$ S. Fisk, The secretary's packet problem, this MAGAzine 61 (1988), 103-105.

4. R. P. Grimaldi, Discrete and Combinatorial Mathematics: An Applied Introduction, Addison-Wesley, Reading, MA, 1990.

5. A. Hald, A History of Probability and Statistics and Their Applications before 1750, Jolın Wiley and Sons, New York, NY, 1989.

6. P. R. de Montmort, Essai d'Analyse sur les Jeux de Hazard, Paris, 1708. Reprinted by Chelsea Publishing, New York, NY, 1980.

7. D. P. Rawlings, A probabilistic approach to some of Euler's number theoretic identities, Trans. Amer. Math. Soc. 350 (1998), 2939-2951.

8. G. R. Sanchez, Swapping hats: A generalization of Montmort's problem, this MAGAzINE 71 (1998), 53-57.

9. P. E. Vernon, The matching method applied to investigations of personality, Psych. Bull. 33 (1936), $149-177$. 